\title{
Key role of poly- $\gamma$-DL-glutamic acid in immune evasion and virulence of Staphylococcus epidermidis
}

\author{
Stanislava Kocianova,, ${ }^{1}$ Cuong Vuong, ${ }^{1}$ Yufeng Yao, ${ }^{1}$ Jovanka M. Voyich, ${ }^{1}$ Elizabeth R. Fischer, ${ }^{2}$ \\ Frank R. DeLeo, ${ }^{1}$ and Michael Otto ${ }^{1}$ \\ ${ }^{1}$ Laboratory of Human Bacterial Pathogenesis and 2Microscopy Core Facility, Rocky Mountain Laboratories, \\ National Institute of Allergy and Infectious Diseases (NIAID), NIH, Hamilton, Montana, USA.
}

\begin{abstract}
Coagulase-negative staphylococci, with the leading species Staphylococcus epidermidis, are the predominant cause of hospital-acquired infections. Treatment is especially difficult owing to biofilm formation and frequent antibiotic resistance. However, virulence mechanisms of these important opportunistic pathogens have remained poorly characterized. Here we demonstrate that $S$. epidermidis secretes poly- $\gamma$-DL-glutamic acid (PGA) to facilitate growth and survival in the human host. Importantly, PGA efficiently sheltered S. epidermidis from key components of innate host defense, namely antimicrobial peptides and neutrophil phagocytosis, and was indispensable for persistence during device-related infection. Furthermore, PGA protected S. epidermidis from high salt concentration, a key feature of its natural environment, the human skin. Notably, PGA was synthesized by all tested strains of $S$. epidermidis and a series of closely related coagulase-negative staphylococci, most of which are opportunistic pathogens. Our study presents important novel biological functions for PGA and indicates that PGA represents an excellent target for therapeutic maneuvers aimed at treating disease caused by $S$. epidermidis and related staphylococci.
\end{abstract}

\section{Introduction}

Over the past decade, Staphylococcus epidermidis has become the most prevalent pathogen involved in nosocomial infections (1). Usually an innocuous commensal microorganism on human skin, this member of the coagulase-negative group of staphylococci can cause severe infection after penetration of the epidermal protective barriers of the human body. In the US alone, S. epidermidis infections on in-dwelling medical devices, which represent the main type of infection with S. epidermidis, cost the public health system approximately $\$ 1$ billion per year (1). Importantly, S. epidermidis is frequently resistant to common antibiotics (1). It is, therefore, essential to find new molecular targets for drug development against S. epidermidis infections. The formation of surface-attached cellular agglomerations, so-called biofilms, is a major determinant of S. epidermidis pathogenicity (2). Biofilm formation is believed to contribute significantly to antibiotic resistance and protection from innate host defense (3). Resistance of S. epidermidis biofilms to some antibiotics might, in part, be due to a status of generally reduced metabolism (4). Yet, by and large, the specific mediators of biofilm resistance and immune evasion in S. epidermidis are unknown.

Poly- $\gamma$-DL-glutamic acid (PGA) is an anionic, extracellular polymer, in which the $\alpha$-amino and $\gamma$-carboxy groups of D- or L-glutamic acid are linked by isopeptide bonds (5). PGA is produced primarily by Bacillus strains but also infrequently by other strains of bacteria, archaebacteria, and some eukaryotes (5). On the whole, the biological role of PGA has remained unclear. Presumably, it serves as an external $\mathrm{C}$ and $\mathrm{N}$ reserve or protects from environmen-

Nonstandard abbreviations used: PGA, poly- $\gamma$-DL-glutamic acid; PIA, polysaccharide intercellular adhesin; PMN, polymorphonuclear leukocyte; TSB, tryptic soy broth. Conflict of interest: The authors have declared that no conflict of interest exists.

Citation for this article: J. Clin. Invest. 115:688-694 (2005)

doi:10.1172/JCI200523523 tal factors such as high osmolarity (5). In Bacillus anthracis, a PGA capsule has a hitherto unique role in sheltering the bacteria from phagocytosis (6). Homologs of the cap locus for PGA biosynthesis are found in a restricted number of human pathogens, including the spirochaete Leptospira interrogans, which causes the waterborne disease leptospirosis (7), and Fusobacterium nucleatum, which causes periodontal disease (8). However, it is unknown whether PGA is produced and plays a role in virulence in these organisms.

Here we demonstrate PGA production in S. epidermidis and related coagulase-negative staphylococci. We constructed an isogenic cap mutant strain of S. epidermidis to analyze the role of PGA in protection from environmental factors and innate host defense and as a virulence determinant in an animal infection model. Our study indicates an important function of PGA during both the commensal and infectious lifestyles of S. epidermidis and presents PGA production as the first widespread mechanism of immune evasion in this principal opportunistic pathogen.

\section{Results}

The S. epidermidis genome contains the genes that code for PGA synthesis. To survive on the human skin and during infection of in-dwelling medical devices, S. epidermidis must have mechanisms to circumvent human innate host defense. Only recently, we discovered that the biofilm exopolysaccharide polysaccharide intercellular adhesin (PIA) protects S. epidermidis from major mechanisms of innate host defense (9). However, PIA is restricted to a subpopulation of S. epidermidis, and therefore, a ubiquitous principle protecting S. epidermidis from innate host defense has remained elusive. We searched the recently published S. epidermidis genome (10) for gene loci potentially involved in such protection. We found that, in contrast to its relative $S$. aureus, S. epidermidis has the cap locus that codes for production of the anionic exopolymer PGA. The S. epidermidis cap genes show high similarity to those of B. anthracis and B. subtilis (Figure 

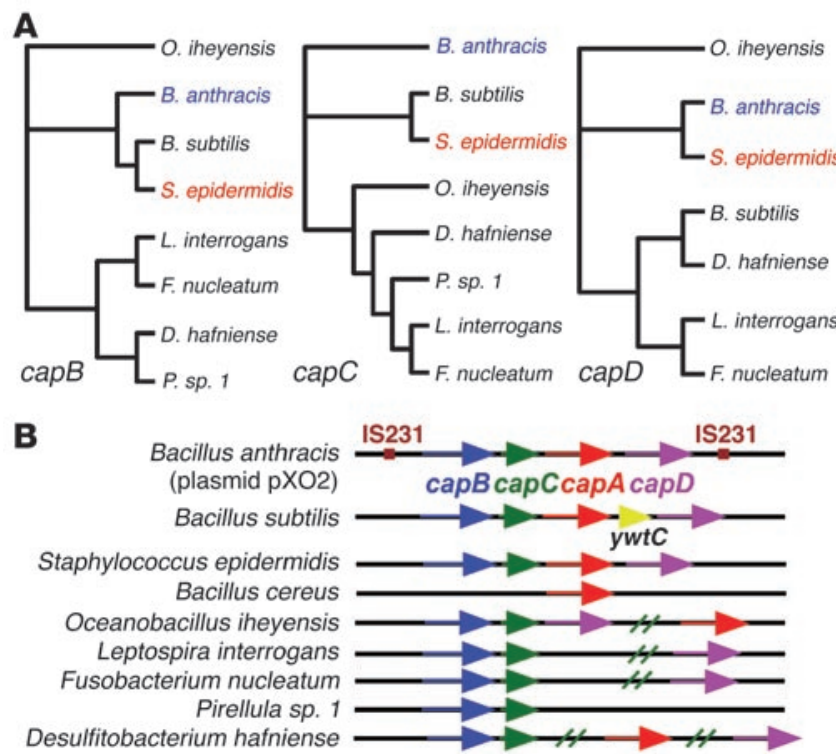

1A). Of note, in contrast to B. subtilis and several human pathogens that have parts of the cap genes, the genetic organization of the cap locus of $B$. anthracis is well conserved in S. epidermidis (Figure 1B).

The S. epidermidis cap gene locus drives production of surface-attached PGA. To analyze the role of the cap genes in S. epidermidis, we constructed a deletion mutant (S. epidermidis $\Delta$ cap), in which the entire cap locus was replaced by a spectinomycin resistance cassette. To complement the mutant, we cloned the cap operon with its natural promoter in a plasmid and transformed the mutant with that plasmid (pRBcap$B C A D$ ). First, we determined PGA production in the mutant, wildtype, and complemented strains. Real-time PCR data indicated that the cap genes in S. epidermidis are expressed (data not shown). Accordingly, immunodetection of PGA in cell surface extracts of

\section{Figure 1}

Molecular genetic comparison of bacteria with genes encoding a putative PGA synthesis machinery. (A) Phylogenetic trees based on sequence comparisons of the capB (amide ligase), capC (unknown function), and capD (depolymerase) genes. CapA is a putative PGA exporter. We have excluded a comparison of capA genes, because capA homologs were not found in all the organisms and comparison of transporters is normally less indicative of phylogenetic relations. Of the microorganisms shown, production of PGA has been demonstrated previously only in $B$. anthracis and $B$. subtilis, and in this study, in S. epidermidis. (B) cap genes and homologs in bacteria for which genetic information is available. The $B$. anthracis cap gene cluster is located on a plasmid and flanked by IS231 insertion sequences. All other genes are located in the bacterial chromosomes.

the wild-type and complemented strains demonstrated that PGA is synthesized by S. epidermidis and attached to the bacterial cell surface (Figure 2A). The PGA production level in the complemented strain was $147 \%$ of that of the wild-type strain. There was no detectable signal in the cap deletion strain or in any samples obtained from culture filtrates (Figure 2A and data not shown). Scanning electron microscopy with immunogold labeling confirmed that PGA is located on the S. epidermidis cell surface (Figure 2B). Next, we purified the immunoreactive material and performed quantitative and qualitative analyses to verify that it constitutes PGA. In contrast to $B$. anthracis PGA, which is entirely composed of D-glutamic acid (11), S. epidermidis PGA consisted of about equal amounts of D- and L-glutamic acid (Figure 2C). Notably, PGA production in S. epidermidis was far less than that reported for $B$. anthracis $\left(1.2 \times 10^{-6} \mathrm{~g} / 1\right.$ for S. epidermidis versus $5-10 \mathrm{~g} / 1$ for $B$. anthracis; ref. 12). Taken together, our data demonstrate that the cap locus in S. epidermidis drives production of a surface-attached form of DL-PGA.

The cap gene locus and PGA production are ubiquitous among S. epidermidis strains. Some virulence factors in staphylococci, such as PIA, are mostly restricted to invasive strains $(13,14)$. In contrast,
A
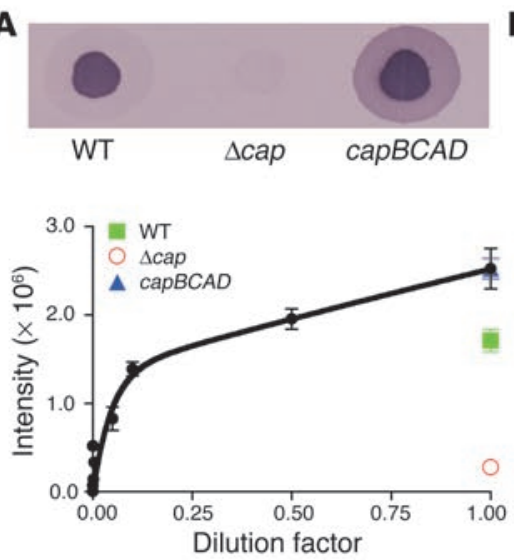

B

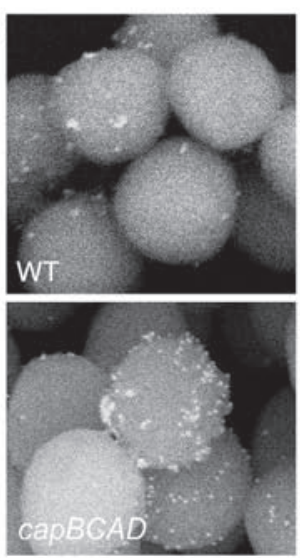

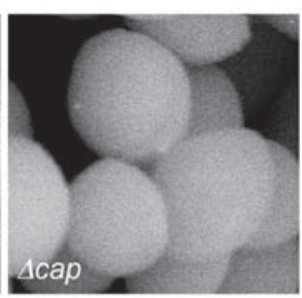

C

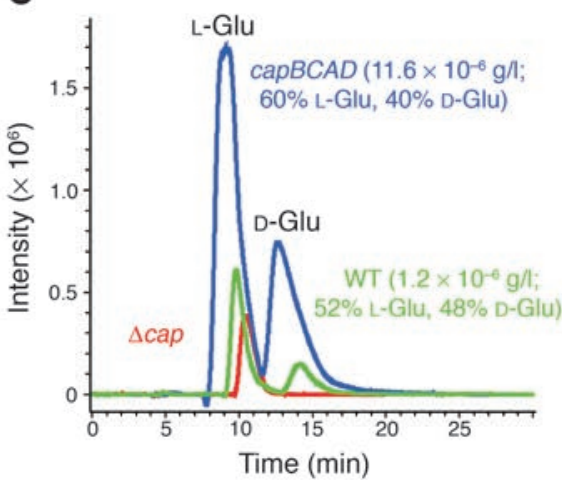

Figure 2

PGA production in S. epidermidis. (A) Relative expression of PGA determined by immuno-dot blot analysis. PGA was extracted from bacterial cell surfaces as described in Methods. A calibration curve was obtained by dilution of the most intensive sample obtained from the PGAoverexpressing complemented strain S. epidermidis $\triangle$ cap (pRBcapBCAD). Results are the mean \pm SEM of 4 experiments for the samples and the mean \pm SEM of 4 different serial dilutions for the calibration curve. A representative blot is shown at the top. (B) Detection of $S$. epidermidis PGA with immunoscanning electron microscopy. PGA was detected with anti-PGA antiserum. (C) Analysis of D-glutamic (D-Glu) and L-glutamic (L-Glu) acid in S. epidermidis PGA by stereoselective chromatography and liquid chromatographic-mass spectrometric detection of glutamic acid. To determine D- and L-glutamic acid amounts, the L-glutamic acid background detected in the cap mutant strain $(\triangle c a p)$ was subtracted from PGA expression strains. (A-C) $\triangle$ cap, isogenic cap deletion strain; capBCAD, complemented strain S. epidermidis $\triangle$ cap (pRBcapBCAD). 


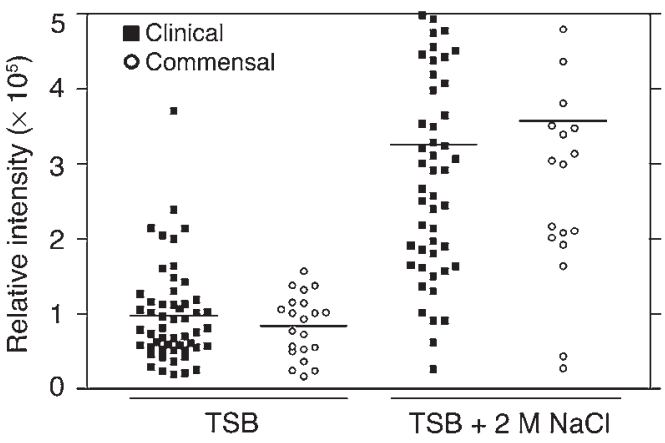

Figure 3

PGA production in S. epidermidis strains. PGA expression in S. epidermidis strains of clinical and commensal origin, and under low and high salt conditions, determined by immuno-dot blot analysis. Cells were grown for 24 hours at $37^{\circ} \mathrm{C}$ with shaking at $200 \mathrm{rpm}$. PGA was then purified as described in Methods. Horizontal bars show the group mean. The membrane background was subtracted from each sample. The experiment, including purification and detection, was repeated twice with very similar results.

all 74 S. epidermidis strains of clinical and commensal origin that we investigated had the cap gene locus as shown by analytical PCR (data not shown) and were positive for PGA by immunodot blot analysis (Figure 3), indicating that PGA production is ubiquitous in S. epidermidis. Remarkably, there was no significant difference in PGA production between commensal and clinical strains (Figure 3). We therefore hypothesized that PGA is important for the survival of $S$. epidermidis as a skin commensal organism and during infection.

$P G A$ contributes to resistance of S. epidermidis to bigh salt concentration. We first determined whether PGA impacts survival of S. epidermidis under conditions found in its natural habitat, i.e., the human skin. The environment on human skin is characterized by high and varying salt concentration. S. epidermidis can withstand very high salt concentrations of up to approximately $2 \mathrm{M} \mathrm{NaCl}$, but the reasons for this extraordinary resistance are not completely clear (13). Growth and viability of the cap mutant strain were significantly impaired at high concentrations of $\mathrm{NaCl}$ (shown for $2 \mathrm{M}$
$\mathrm{NaCl}$ in Figure 4, A and B), whereas growth at physiological salt concentration was similar for the wild-type and mutant strains (data not shown). Furthermore, quantitative real-time PCR analysis using a $c a p B$ probe (Figure 4C) and immuno-dot blots (data not shown) demonstrated that $S$. epidermidis upregulates cap expression in response to high $\mathrm{NaCl}$ concentration. Additionally, in most strains of our collection, PGA production was greater at high $\mathrm{NaCl}$ concentration (Figure 3). These data suggest that PGA contributes to survival of S. epidermidis on human skin.

S. epidermidis PGA protects from key components of innate host defense. Next, we investigated whether PGA contributes to S. epidermidis virulence. Unlike its more aggressive relative S. aureus, S. epidermidis does not have a large arsenal of virulence factors (1). Rather, it causes relatively silent, chronic infections during which resistance against attacks by the innate immune system is of special importance to bacterial survival (1). Therefore, we analyzed whether cap expression in S. epidermidis affects resistance to key components of innate host defense against bacterial infections, namely antibacterial peptides and neutrophil phagocytosis. The cap mutant strain had significantly reduced resistance to 2 representative antibacterial peptides from human skin and neutrophil specific granules, LL-37 and human $\beta$-defensin 3 (Figure 5, A and B) and significantly increased susceptibility to phagocytosis by human neutrophils (Figure 5C). The phagocytosis rate was $42 \%$ higher with the mutant strain compared to the wild-type strain, which is a relatively large difference compared with what we observe with other single pathogen factors. These findings indicate that PGA plays a critical role in protecting $S$. epidermidis from the microcidal effects of innate host defense components.

PGA is indispensable for S. epidermidis persistence on in-dwelling medical devices in an animal infection model. The role of PGA in protection from innate host defense components suggested that PGA facilitates pathogen survival during S. epidermidis infection. To test this hypothesis, we compared persistence of wild-type and isogenic cap mutant strains in a mouse model of subcutaneous catheter infection. Importantly, biofilm formation on plastic material and intercellular aggregation in vitro did not differ between wild-type and cap mutant strains (data not shown), indicating that colonization and persistence in the animal model were not due to differences in the physicochemical interaction with the catheter
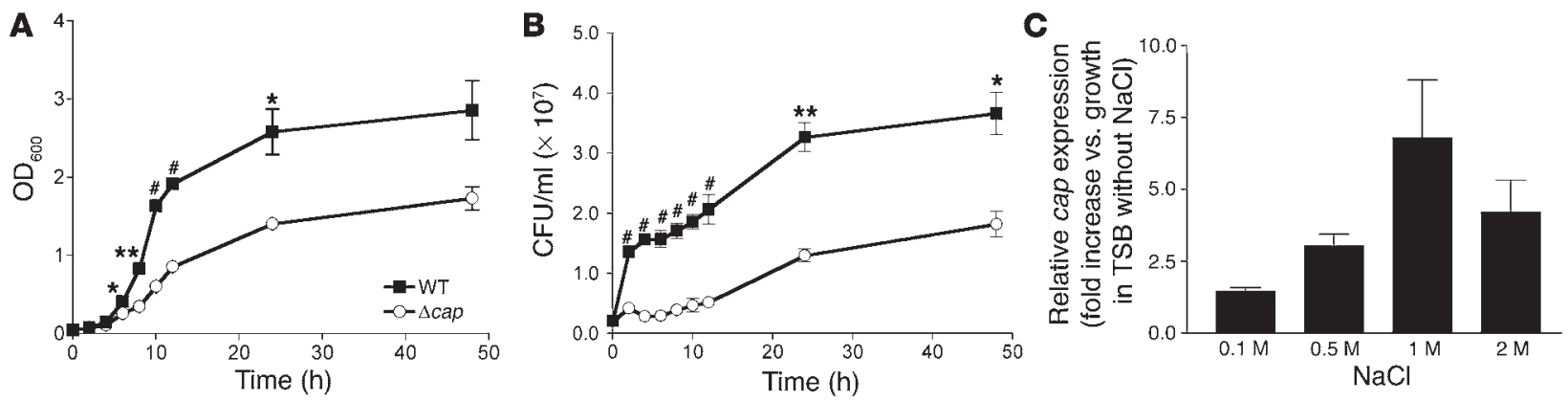

\section{Figure 4}

Role of PGA in osmoprotection and inducibility of cap expression by $\mathrm{NaCl}$. (A) Growth $\left(\mathrm{OD}_{600}\right)$ and (B) viability (CFU) of wild-type and cap mutant strains in Luria-Bertani medium supplemented with $2 \mathrm{M} \mathrm{NaCl}$. Bacteria were inoculated from an overnight preculture $(1: 1,000)$ and grown in flasks at $37^{\circ} \mathrm{C}$ with shaking at $200 \mathrm{rpm}$. Values are the mean \pm SEM of 3 experiments. ${ }^{*} P<0.05 ;{ }^{* *} P<0.01$; ${ }^{\#} P<0.001$ (wild-type versus mutant strain). (C) Quantitative real-time PCR analysis of $\mathrm{NaCl}$ inducibility of cap expression. Bacteria were grown as in (A) with the indicated concentrations of $\mathrm{NaCl}$. Cells were harvested after 6 hours of growth, RNA was isolated, and real-time PCR was performed using a cap $B$ probe. Values are the mean \pm SEM of 3 experiments. 

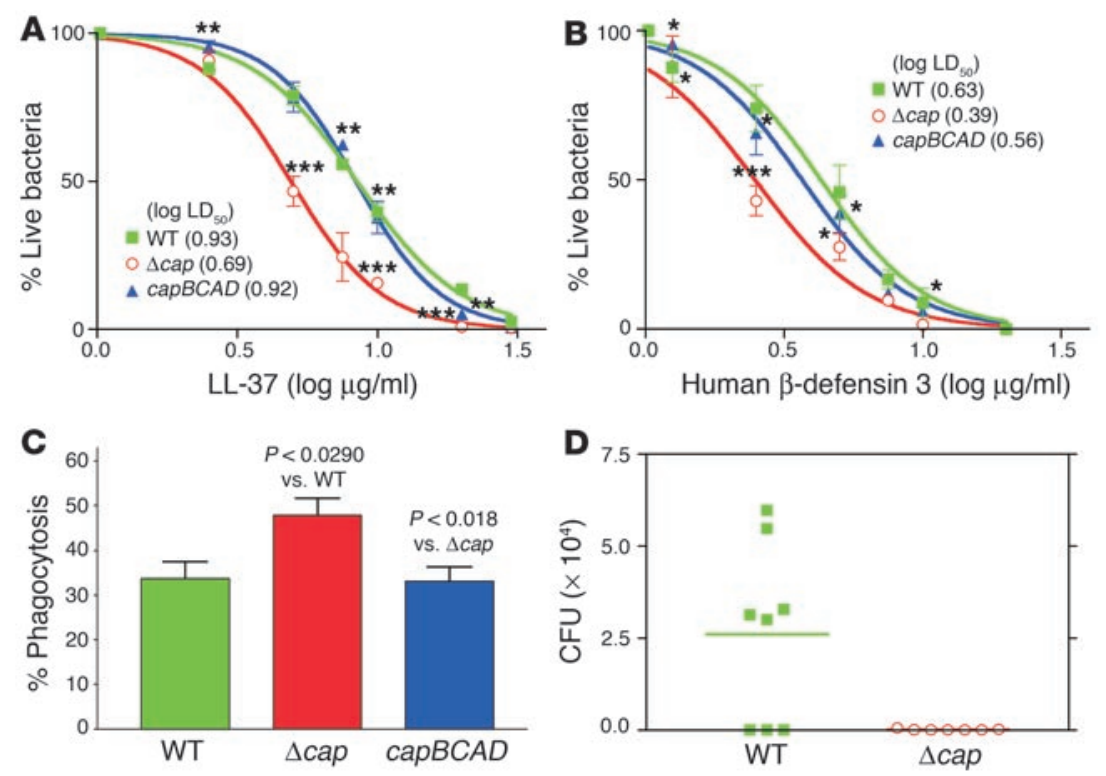

Figure 5

Role of PGA in immune evasion and virulence of S. epidermidis. (A and B) Resistance to cationic antimicrobial peptides. Washed $S$. epidermidis cells (approximately $10^{5}$ ) were incubated with LL-37 (A) or human $\beta$-defensin $3(\mathbf{B})$ in various concentrations for 2 hours at $37^{\circ} \mathrm{C}$. Thereafter, $\mathrm{S}$. epidermidis survivor cells were counted by plating. Results are shown as dose-response curves. The log $L_{50}$ values for all strain/peptide combinations are given in the key. Statistical analyses are for each peptide concentration. Values of significance were calculated against the wild-type (for $\triangle$ cap) and $\triangle$ cap (for capBCAD) strains. (C) Resistance to neutrophil phagocytosis. Phagocytosis by human neutrophils was determined after 30 minutes of incubation with S. epidermidis at a ratio of 20 bacteria per PMN. (D) Mouse model of subcutaneous device-related infection. Catheter pieces with equal amounts of adhered $S$. epidermidis cells $\left(2 \times 10^{5}\right)$ were placed under the dorsum of the animals. CFU on implanted devices 1 week after infection were counted. The horizontal bar shows the group mean. ${ }^{*} P<0.05 ;{ }^{* \star} P<0.01$; ${ }^{* \star *} P<0.001$.

material. Most mice (5 of 8) infected with the wild-type strain had significant numbers of bacteria (approximately $3 \times 10^{4}-6 \times 10^{4}$ CFU) on implanted catheters after 1 week of infection, whereas all 7 mice infected with the cap mutant strain had completely cleared the infection (Figure 5D). This pronounced difference indicates that PGA is a key factor for the success of S. epidermidis in deviceassociated infection, which represents the predominant type of disease caused by this organism.

A group of coagulase-negative staphylococci related to S. epidermidis also produces $P G A$. We know from publicly available genome sequencing data that the cap locus is absent from $S$. aureus. To investigate whether other staphylococcal species have the genetic information for PGA production, we tested a series of staphylococcal strains for the presence of the cap genes by DNA-DNA hybridization using capB and $c a p D$ probes and a cocktail of probes from all 4 cap genes. Of 22 strains, representing 16 different species and subspecies, genomic DNA from 11 strains hybridized with the probes (Table 1). Except for S. saprophyticus strains, all strains with a signal in the Southern blot also produced surface-attached PGA, which was demonstrated using anti-PGA antisera (Table 1). Although there are some intraspecies differences, it is remarkable that all phylogenetically related members of the $S$. epidermidis group $(S$. epidermidis, S. capitis, S. warneri, S. saccharolyticus, S. caprae, S. hominis, and S. haemolyticus) (15) have the ability to produce PGA, whereas it is far less distributed among other species (Table 1).

\section{Discussion}

We are only beginning to understand strategies used by pathogenic bacteria to evade the efficient bactericidal mechanisms of innate host defense (16). To survive in its natural habitat, i.e., on human skin, S. epidermidis needs to protect itself from antibacterial peptides of human epithelial cells. Furthermore, to persist during human infection, it must have efficient protection from ingestion by neutrophils and associated bactericidal mechanisms. Here we present PGA as the first widespread mechanism of immune evasion in S. epidermidis. Notably, we demonstrate a hitherto unknown role for PGA in resistance to cationic antimicrobial peptides. Further, PGA was critical for the establishment of chronic infection by $S$. epidermidis. The difference in persistence of the cap mutant and wild-type strains was more pronounced than with any other virulence determinant of $S$. epidermidis that has been investigated by an animal infection model. Inasmuch as PGA production in S. epidermidis is approximately 1 million times lower than in $B$. anthracis, one can conclude that the efficiency of PGA-mediated protection from innate host defense in the case of S. epidermidis is extraordinary. The mechanism of protection is unclear. Presumably, it is based on the strong anionic properties of PGA. For example, PGA may bind antibacterial peptides, which are usually cationic (17), and thus prevent them from reaching their target, the cytoplasmic membrane. However, protection by PGA may also be mediated, for example, by binding of PGA to other surface polymers, which might enhance the protective effect.

Except for the role that PGA has in protecting $B$. anthracis from phagocytosis, the biological function of PGA, particularly in noninfectious organisms, has remained speculative. It is known that several halophilic bacteria synthesize PGA (5). Therefore, it has been assumed that PGA serves to protect from high osmolarity. In our study, we show for the first time that PGA contributes to resistance against high salt concentration. It has been speculated that binding of water molecules by negatively charged PGA protects bacteria from dehydration (18). However, the low level of PGA production suggests that mechanisms other than direct binding of water by PGA might be involved in the protective effect in S. epidermidis.

Importantly, PGA production did not affect the aggregation behavior or biofilm formation of S. epidermidis, demonstrating that our observations are not due to secondary effects of cell aggregation. However, recent results that we obtained by microarray analysis of gene expression in S. epidermidis biofilms indicated that the cap locus is upregulated in biofilms (4). PGA may thus contribute to the overall resistance of S. epidermidis biofilms to innate immunity, although it does not appear to directly influence biofilm formation.

The S. epidermidis cap locus shows high similarity to that of $B$. anthracis. The genetic information for PGA production in $B$. anthracis is located on a plasmid (pXO2) $(19,20)$ and shows high genetic mobility (21). It might have been transferred between $B$. anthracis and staphylococci and from one strain of Staphylococcus to another. Coinfection of humans or human ancestors may represent a 
Table 1

PGA production in staphylococci

\section{Strain}

S. epidermidis group

S. capitis subsp. capitis

$S$. capitis subsp. ureolyticus

S. caprae

S. haemolyticus

S. warneri

S. warneri

S. saccharolyticus

S. hominis subsp. hominis

$S$. hominis subsp. hominis

Other staphylococci

S. schleiferi subsp. schleiferi

S. pulveri

S. simulans

S. simulans

S. simulans

S. xylosus

S. xylosus

S. saphrophyticus

S. saphrophyticus

S. cohnii subsp. cohnii

S. cohnii subsp. urealyticum

$S$. lugdunensis

$S$. intermedius

$S$. aureus
ATCC no.

ATCC 27840

ATCC 49324

ATCC 51548

ATCC 29970

ATCC 17917

ATCC 49518

ATCC 14953

ATCC 25615

ATCC 27844

ATCC 43808

ATCC 51698

ATCC 31432

ATCC 700576

ATCC 27848

ATCC 49148

ATCC 29966

ATCC 35552

ATCC 15305

ATCC 29972

ATCC 49328

ATCC 43809

ATCC 49052

All strains with known

genome sequence
PGA

production $^{A}$

Presence of $\operatorname{cap} B$

and $\operatorname{cap}$ genes ${ }^{\mathrm{B}}$

$\begin{array}{ll}+ & + \\ + & + \\ + & + \\ + & + \\ + & + \\ - & + \\ + & + \\ + & + \\ - & \end{array}$

+
+
+
+
+
+
+

$\begin{array}{ll}- & - \\ - & -\end{array}$

$-$

$+$

$-$

$-$

$-$

$-$

$-$

$-$

$-$

$+$

ND

(1)

ABy immuno-dot blot analysis. BBy Southern blot analysis. ND, not determined.

likely scenario for these events. Of note, most of the staphylococcal strains that produce PGA are opportunistic human pathogens. Consistent with its role in S. epidermidis, PGA might thus contribute to survival of those strains in the human host, although this notion remains to be demonstrated.

In conclusion, PGA appears to play a key role for the survival of S. epidermidis in the human host during colonization and infection. Remarkably, though produced in very small amounts, PGA contributes significantly to the pathogenicity of S. epidermidis, which underscores the efficiency of PGA-mediated protection. PGA may represent an excellent target for drug or vaccine development against infection with $S$. epidermidis and possibly other staphylococcal pathogens. Anti-PGA antibodies have been suggested for the treatment and prophylaxis of $B$. anthracis infections. Although PGA has a generally low immunogenicity, recent studies have shown that anti-PGA antibodies efficiently protect from anthrax infection in animal models (22-25). Our data indicate that they might also be valuable for the treatment of chronic infections by $S$. epidermidis and certain other pathogenic staphylococci.

\section{Methods}

Bacterial strains, growth conditions, and basic molecular biology methods. The clinical isolate S. epidermidis 1457 (26) was used in this study. Bacteria were grown in tryptic soy broth (TSB) unless otherwise noted. Antibiotics were used at the following concentrations: chloramphenicol, $10 \mu \mathrm{g} / \mathrm{ml}$; spectinomycin, $100 \mu \mathrm{g} / \mathrm{ml}$; and ampicillin, $100 \mu \mathrm{g} / \mathrm{ml}$. Cultures were incubated at $37^{\circ} \mathrm{C}$ with shaking at $200 \mathrm{rpm}$. DNA manipulation, isolation of plasmid DNA, and transformation of Escherichia coli were performed using standard procedures. Staphylococcal plasmid DNA was prepared with the Qiagen Plasmid Midi Kit as described (27). S. epidermidis was transformed by electroporation as described (28). PCRs were performed with Ready-To-Go PCR Beads (Amersham Biosciences) as recommended by the manufacturer. DNA was sequenced using Big Dye Terminator cycle sequencing (version 3.0) on an ABI3700 sequencer (Applied Biosystems). Nucleotide sequences were analyzed using the program Vector NTI Suite (InforMax). Primers for DNA amplifications by PCR (Table 2) were purchased from Sigma Genosys. The 74 strains used for epidemiological studies were obtained from N. El Solh (Institut Pasteur, Paris, France) and are predominantly nonclonal (29).

Real-time PCR. RNA isolation was performed using a FastPrep BLUE Kit (Q-BioGene Inc.). Oligonucleotide primers and probes were designed using Primer Express 2.0 software (Applied Biosystems). The probe used for analysis of cap expression was located within the $c a p B$ gene. TaqMan analysis was performed in a 384-well MicroAmp Optical using a 7900 Sequence Detector (Applied Biosystems). Standard curves were determined with purified chromosomal template DNA at concentrations ranging from $0.001 \mathrm{ng} / \mathrm{ml}$ to $10 \mathrm{ng} / \mathrm{ml}$. Assays were performed in triplicate using cDNA samples, and 16S rRNA as control, with a standard cycle protocol.

Southern blot analysis. Equal amounts of EcoRV-digested genomic DNA were separated by gel electrophoresis on a $0.7 \%$ agarose gel and visualized by ethidium bromide staining. DNA was transferred onto a nylon membrane (GE Osmonics Labstore) and probed with digoxigenin-labeled PCR product amplified from the $c a p B$ or capD genes of S. epidermidis 1457 or with a cocktail of probes amplified from the cap $A, c a p B, c a p C$, and cap $D$ genes. Prehybridization, hybridization, posthybridization, and immunologic detection were performed as described in the technical update accompanying the nonradioactive DNA Labeling and Detection Kit (Roche Applied Science). For immunologic detection, the membrane was incubated with a 1:5,000 dilution of sheep anti-digoxigenin Fab fragments conjugated to alkaline phosphatase. Probe-target hybrids were detected using the chromogenic substrate nitroblue tetrazolium/ 5-bromo-4-chloro-3-indolyl phosphate.

Immuno-dot blot assay. Surface-attached PGA was released from the cell surface by boiling bacteria for 30 minutes at $100^{\circ} \mathrm{C}$ or by autoclaving. PGA was then purified by acid precipitation as described (12). Aliquots $(3 \mu \mathrm{l})$ of the samples were spotted on a nitrocellulose membrane, air-dried, and PGA was detected with anti-PGA antiserum using a scanner and Total Lab Version 2003 software (Nonlinear USA). The assay was calibrated by serial dilution of the most intensive sample. The value detected in the wild-type strain was set to $100 \%$ and production values in the other strains were expressed relative to that value. Anti-PGA antiserum (kindly provided by R. Schneerson, National Institute of Child Health \& Human Development, NIH, Bethesda, Maryland, USA) was developed against PGA from Bacillus pumilus. For Staphylococcus strains other than S. epidermidis, a strain with a signal higher than that of the background of the S. epidermidis $\Delta$ cap mutant was considered a PGA producer.

Construction of an isogenic cap deletion mutant and cap-complementing vector. To delete capBCAD in S. epidermidis 1457, PCR-amplified regions flanking the cap locus and a spectinomycin resistance cassette were cloned into plasmid pBT2 (30), yielding plasmid pBT $\Delta c a p$, which was used for allelic replacement as described (31). The proper integration of the resistance gene marker $s p c$ was verified by direct sequencing of the genomic DNA at the borders of the PCR-derived regions. Lack of cap transcript in the cap mutant strain was verified by real-time PCR (data not shown). S. epidermidis 1457 in which 
Table 2

Oligonucleotides used in this study

\begin{tabular}{|c|c|}
\hline \multicolumn{2}{|c|}{ For TaqMan analysis of cap expression } \\
\hline capF & CATGAAGCTGAGAATGCACTTGTATT \\
\hline CapProbe & AACATCCACGGCCCGG \\
\hline capR & СTATCCСTTCTATGAATTCCGCTATT \\
\hline \multicolumn{2}{|c|}{ For allelic replacement of the cap locus } \\
\hline CapEco & CCACCAATATCCGTTGCTTGAGGTGCAGCAGAATTCATCC \\
\hline CapBam & GGGTATTGGTACACCAGGTGGGAACAAGGATCCAACAATTC \\
\hline CapSal & GCGCAACACACGCTATAATGAGTAACAGTCGACTTTACCTCTC \\
\hline CapHind & CATGTCTTTACCATTTAAGCTTCCAATAAGTATAAATGCGAGG \\
\hline \multicolumn{2}{|c|}{ For construction of complementation vectors } \\
\hline capBam2 & САATCATCATACTACTTCTTTCATTCATTGGATCCGCTTACAC \\
\hline capXba & GACTTCTCCATACCTCTCCTCCTCTAGACGTAATATC \\
\hline \multicolumn{2}{|c|}{ To confirm lack of cap expression in the cap mutant strain by real-time PCR } \\
\hline CAP1 & CCCTTCTATGAATTCCGCTATTCTACCACCCGGGCCGTGG \\
\hline CAP2 & GACGTCATACCAGAATCATATTTACGGAAGTTCG \\
\hline C2P1 & GATATTGATCACAACTCACC \\
\hline C2P5 & GCCATTATCTGTGTTTTCAC \\
\hline \multicolumn{2}{|c|}{ For amplification of the capB probe (Southern blot) } \\
\hline CapSB2 & ATGTGATGGAAGACCATATGGATGTCTTAGGACCGACACT \\
\hline CapSB3 & CGCTTTTGTAGACTGCGGTTCATTAGCAGCGAATGCATTA \\
\hline CapSB-D3 & ATCCTTGAAGCATTAATGTGCCTCCTAAAGGATTAGGTGC \\
\hline CapSB-D4 & CTCATTCATCAGGACTAGGAGGTGGCGGTGCGACACTTAC \\
\hline CapSB-A1 & AATGACATCTGCACCAGCATTCGCTAACGCATGTGCATAT \\
\hline CapSB-A2 & GAGAGACAAAGATAGCATTCGTATCACCTATTTAGGTAAC \\
\hline CapSB-C1 & GACAACACCTATACCTGAAACTTCAACCATTTCAAATGGGGTC \\
\hline CapSB-C2 & GCTGAGAAATTTGGGATTAATCCAGCAGGGTTAGTCGTTC \\
\hline
\end{tabular}

tom microtiter plates. Plates were incubated for 30 minutes at $37^{\circ} \mathrm{C}$ and the degree of phagocytosis was determined by flow cytometry.

Peptide bacterial killing assays. S. epidermidis cultures were harvested, washed with PBS buffer, and resuspended in $10 \mathrm{mM}$ sodium phosphate buffer ( $\mathrm{pH}$ 7.0). Bacterial killing assays were performed using a final concentration of approximately $10^{5}$ S. epidermidis cells in each sample. Antimicrobial peptides were dissolved in the following solutions: human $\beta$-defensin 3, $10 \mathrm{mM}$ acetic acid, and LL-37, $10 \%$ acetonitrile with $0.1 \%$ trifluoroacetic acid. The bacteria were exposed to a range of antimicrobial peptide concentrations $(0,5,10,20,30$, and $40 \mu \mathrm{g} / \mathrm{ml})$. An equal volume of the respective peptide dilution buffer was applied to control samples. Samples were incubated at $37^{\circ} \mathrm{C}$ for 2 hours and appropriate dilution series of the samples were plated on TSB agar. Survivor S. epidermidis cells were enumerated after 24 hours of incubation at $37^{\circ} \mathrm{C}$. The percentage of killed S. epidermidis was calculated using the formula $\left(1-\left[\mathrm{CFU}_{\text {peptide }} / \mathrm{CFU}_{\text {control }}\right]\right) \times 100$.

Scanning immunoelectron microscopy. Fifty-microliter aliquots of $S$. epidermidis cultures were washed with PBS buffer. Cells were resuspended in $200 \mu \mathrm{l}$ of anti-PGA antiserum and incubated at $37^{\circ} \mathrm{C}$ with agitation at $400 \mathrm{rpm}$ for 12 hours. Samples were washed with PBS and pellets were subsequently incubated with goat anti-rabbit IgG conjugated with $20 \mathrm{~nm}$ of gold (BB International) at $37^{\circ} \mathrm{C}$ with agitation at $400 \mathrm{rpm}$ for 2 hours. Following antibody labeling, the cell suspensions were attached to coverslips, fixed with $2.5 \%$ glutaraldehyde in $0.1 \mathrm{M}$ sodium cacodylate, and post-fixed with $1 \%$ osmium tetroxide in $0.1 \mathrm{M}$ sodium cacodylate. Samples were washed with distilled water, dehydrated in a graded ethanol series, criticalpoint dried under $\mathrm{CO}_{2}$ with a Bal-Tec model cpd 030 drier (Balzers),

capBCAD was deleted was named S. epidermidis $\triangle$ cap. To complement for cap $B C A D$ in S. epidermidis $\triangle c a p, c a p B C A D$ genes were cloned into plasmid pRB474 (30). The resulting plasmid was named pRBcapBCAD.

Purification of $P G A$ and detection of $D$ - and $L$-glutamic acid. Cultures were grown in TSB medium supplemented with $1 \mathrm{M} \mathrm{NaCl}$ to induce PGA production. PGA was first purified as described above. Then, PGA samples were further purified by ion exchange chromatography using a RESOURCE Q $6 \mathrm{ml}$ column (Amersham Biosciences) on an AKTA Purifier 10 (Amersham Biosciences) and a gradient from $0.2 \%$ acetic acid to $0.2 \%$ acetic acid $/ 1 \mathrm{M}$ $\mathrm{NaCl}$ in 20 column volumes at a flow rate of $5 \mathrm{ml} / \mathrm{min}$. Fractions with positive reaction in an immuno-dot blot were combined, dialyzed against water, lyophilized, resuspended in $6 \mathrm{M} \mathrm{HCl}$, and hydrolyzed at $110^{\circ} \mathrm{C}$ for 24 hours. Samples were lyophilized again and dissolved in $200 \mu \mathrm{l}$ of water. Ten microliters of the samples were then injected onto a Chirobiotic T column (Astec) using $0.1 \%$ triethylammonium acetate ( $\mathrm{pH} 4.0$ ) in $80 \%$ ethanol as elution buffer at a flow rate of $0.4 \mathrm{ml} / \mathrm{min}$. Chromatography was performed using an Agilent 1100 series HPLC connected to a VL Trap mass spectrometer. The extracted ion chromatograms at $146 \mathrm{Da}$, the mass of glutamic acid, were used to determine the amounts of D- and L-glutamic acid by peak integration in comparison to pure D- and L-glutamic acid.

Isolation of human polymorphonuclear leukocytes and phagocytosis experiments. Human polymorphonuclear leukocytes (PMNs) were isolated from heparinized venous blood of healthy individuals with a standard method (32). All studies were performed in accordance with a protocol approved by the Institutional Review Board for Human Subjects of NIAID. Cell preparations contained approximately 99\% PMNs and all reagents used contained $<25.0$ $\mathrm{pg} / \mathrm{ml}$ endotoxin. Phagocytosis of S. epidermidis by human PMNs was analyzed by flow cytometry with a previously described method (32). Briefly, bacteria were cultured to stationary growth phase, washed in PBS, and labeled with fluorescein-5-isothiocyanate for 15 minutes. PMNs $\left(10^{6} / 100 \mu \mathrm{l}\right)$ and bacteria $\left(2 \times 10^{7} / 100 \mu \mathrm{l}\right)$ were combined in wells of serum-coated 96 -well round-bot- mounted on aluminum studs, and sputter-coated with 100 angstroms of chromium in a model IBS/TM200S ion beam sputterer (South Bay Technologies) prior to viewing at $10 \mathrm{kV}$ on a Hitachi S-4500 field emission scanning electron microscope (Hitachi) in backscatter imaging mode.

Murine model of device-related infection. Female Balb/c mice were used in a model of subcutaneous implanted device-related infection as described (33). Two catheter pieces of 1-cm length were placed under the skin of the dorsum of each animal. CFUs on catheters were counted before insertion and were in the range of $2 \times 10^{5}$ on all implanted catheters. $\mathrm{CFU}$ on excised catheters and surrounding tissues were counted after 1 week of infection. All studies were performed in accordance with a protocol approved by the Animal Care and Use Committee of Rocky Mountain Laboratories, NIAID.

Statistics and DNA sequence analysis. Statistical analysis was performed using GraphPad Prism version 4.0. DNA sequences were compared using Clustal W software.

The authors would like to thank Rachel Schneerson for providing anti-PGA antisera, Nevine El Solh for S. epidermidis strains, Aaron B. Carmody for technical assistance, and Donald Gardner, Michael Parnell, and Ralph Larson for help with animal studies.

Received for publication October 1, 2004, and accepted in revised form December 7, 2004.

Address correspondence to: Michael Otto, Laboratory of Human Bacterial Pathogenesis, Rocky Mountain Laboratories, National Institute of Allergy and Infectious Diseases, The National Institutes of Health, 903 South 4th Street, Hamilton, Montana 59840, USA. Phone: (406) 363-9283; Fax: (406) 375-9677; E-mail: motto@niaid.nih.gov.

\section{Acknowledgments}


1. Vuong, C., and Otto, M. 2002. Staphylococcus epidermidis infections. Microbes Infect. 4:481-489.

2. Götz, F. 2002. Staphylococcus and biofilms. Mol. Microbiol. 43:1367-1378.

3. Costerton, J.W., Stewart, P.S., and Greenberg, E.P. 1999. Bacterial biofilms: a common cause of persistent infections. Science. 284:1318-1322.

4. Yao, Y., Sturdevant, D.E., and Otto, M. 2005. Genome-wide analysis of gene expression in Staphylococcus epidermidis biofilms: insights into $S$ epidermidis biofilm pathophysiology and the role of phenol-soluble modulins in biofilm formation. J. Infect. Dis. 191:289-298.

5. Oppermann-Sanio, F.B., and Steinbüchel, A. 2002 Occurrence, functions and biosynthesis of polyamides in microorganisms and biotechnological production. Naturwissenschaften. 89:11-22.

6. Little, S.F., and Ivins, B.E. 1999. Molecular pathogenesis of Bacillus anthracis infection. Microbes Infect. 1:131-139.

7. Ren, S.X., et al. 2003. Unique physiological and pathogenic features of Leptospira interrogan revealed by whole-genome sequencing. Nature. 422:888-893.

8. Kapatral, V., et al. 2002. Genome sequence and analysis of the oral bacterium Fusobacterium nucleatum strain ATCC 25586. J. Bacteriol. 184:2005-2018.

9. Vuong, C., et al. 2004. Polysaccharide intercellular adhesin (PIA) protects Staphylococcus epidermidis against major components of the human innate immune system. Cell. Microbiol. 6:269-275.

10. Zhang, Y.Q., et al. 2003. Genome-based analysis of virulence genes in a non-biofilm-forming Staphylococcus epidermidis strain (ATCC 12228). Mol. Microbiol. 49:1577-1593.

11. Ashiuchi, M., and Misono, H. 2002. Biochemistry and molecular genetics of poly-gamma-glutamate synthesis. Appl. Microbiol. Biotechnol. 59:9-14.

12. Hanby, W.E., and Rydon, H.N. 1946. The capsular substance of Bacillus anthracis. Biochemistry. 40:297-307

13. Otto, M. 2004. Virulence factors of the coagulase- negative staphylococci. Front. Biosci. 9:841-863.

14. Galdbart, J.O., Allignet, J., Tung, H.S., Ryden, C. and El Solh, N. 2000. Screening for Staphylococcus epidermidis markers discriminating between skinflora strains and those responsible for infections of joint prostheses. J. Infect. Dis. 182:351-355.

15. Saruta, K., et al. 1997. Rapid identification and typing of Staphylococcus aureus by nested PCR amplified ribosomal DNA spacer region. FEMS Microbiol. Lett. 146:271-278

16. Hornef, M.W., Wick, M.J., Rhen, M., and Normark, S. 2002. Bacterial strategies for overcoming host innate and adaptive immune responses. Nat. Immunol. 3:1033-1040.

17. Hancock, R.E., and Diamond, G. 2000. The role of cationic antimicrobial peptides in innate host defences. Trends Microbiol. 8:402-410.

18. Hezayen, F.F., Rehm, B.H., Eberhardt, R., and Steinbüchel, A. 2000. Polymer production by two newly isolated extremely halophilic archaea: application of a novel corrosion-resistant bioreactor. Appl. Microbiol. Biotechnol. 54:319-325.

19. Green, B.D., Battisti, L., Koehler, T.M., Thorne, C.B., and Ivins, B.E. 1985. Demonstration of a capsule plasmid in Bacillus anthracis. Infect. Immun. 49:291-297.

20. Makino, S., Sasakawa, C., Uchida, I., Terakado, N., and Yoshikawa, M. 1988. Cloning and $\mathrm{CO}_{2}$-dependent expression of the genetic region for encapsulation from Bacillus anthracis. Mol. Microbiol. 2:371-376.

21. Green, B.D., Battisti, L., and Thorne, C.B. 1989. Involvement of Tn4430 in transfer of Bacillus anthracis plasmids mediated by Bacillus thuringiensis plasmid pXO12. J. Bacteriol. 171:104-113.

22. Leppla, S.H., Robbins, J.B., Schneerson, R., and Shiloach, J. 2002. Development of an improved vaccine for anthrax. J. Clin. Invest. 110:141-144. doi:10.1172/JCI200216204.

23. Schneerson, R., et al. 2003. Poly(gamma-Dglutamic acid) protein conjugates induce IgG antibodies in mice to the capsule of Bacillus anthracis: a potential addition to the anthrax vaccine. Proc. Natl. Acad. Sci. U. S. A. 100:8945-8950.

24. Kozel, T.R., et al. 2004. mAbs to Bacillus anthracis capsular antigen for immunoprotection in anthrax and detection of antigenemia. Proc. Natl. Acad. Sci. U. S. A. 101:5042-5047.

25. Rhie, G.E., et al. 2003. A dually active anthrax vaccine that confers protection against both bacilli and toxins. Proc. Natl. Acad. Sci. U. S. A. 100:10925-10930.

26. Mack, D., et al. 1994. Characterization of transposon mutants of biofilm-producing Staphylococcus epidermidis impaired in the accumulative phase of biofilm production: genetic identification of a hexosamine-containing polysaccharide intercellular adhesin. Infect. Immun. 62:3244-3253.

27. Vuong, C., Gerke, C., Somerville, G.A., Fischer, E.R., and Otto, M. 2003. Quorum-sensing control of biofilm factors in Staphylococcus epidermidis. J. Infect. Dis. 188:706-718.

28. Augustin, J., et al. 1992. Genetic analysis of epidermin biosynthetic genes and epidermin-negative mutants of Staphylococcus epidermidis. Eur. J. Biochem. 204:1149-1154.

29. Galdbart, J.O., Morvan, A., Desplaces, N., and el Solh, N. 1999. Phenotypic and genomic variation among Staphylococcus epidermidis strains infecting joint prostheses. J. Clin. Microbiol. 37:1306-1312.

30. Brückner, R. 1997. Gene replacement in Staphylococcus carnosus and Staphylococcus xylosus. FEMS Microbiol. Lett. 151:1-8.

31. Vuong, C., Götz, F., and Otto, M. 2000. Construction and characterization of an agr deletion mutant of Staphylococcus epidermidis. Infect. Immun. 68:1048-1053.

32. Voyich, J.M., et al. 2003. Genome-wide protective response used by group A Streptococcus to evade destruction by human polymorphonuclear leukocytes. Proc. Natl. Acad. Sci. U. S. A. 100:1996-2001.

33. Kadurugamuwa, J.L., et al. 2003. Direct continuous method for monitoring biofilm infection in a mouse model. Infect. Immun. 71:882-890. 